\title{
BMJ Global Health Integrated digital system for community engagement and community-based surveillance during the 2014-2016 Ebola outbreak in Sierra Leone: lessons for future health emergencies
}

\author{
Mohamed F Jalloh (D) , ${ }^{1,2}$ Paul Sengeh, ${ }^{2}$ Nyuma James, ${ }^{2}$ Saiku Bah, ${ }^{3}$ \\ Mohammad B Jalloh, ${ }^{2}$ Katharine Owen, ${ }^{4}$ Samuel Abu Pratt, ${ }^{2}$ Allan Oniba, ${ }^{4}$ \\ Musa Sangarie, ${ }^{5}$ Samuel Sesay, ${ }^{6}$ Jamie Bedson $^{3}$
}

\begin{abstract}
To cite: Jalloh MF, Sengeh $P$, James N, et al. Integrated digital system for community engagement and communitybased surveillance during the 2014-2016 Ebola outbreak in Sierra Leone: lessons for future health emergencies. BMJ Global Health 2020;5:e003936. doi:10.1136/ bmjgh-2020-003936
\end{abstract}

Handling editor Seye Abimbola

- Additional material is published online only. To view, please visit the journal online (http://dx.doi.org/10.1136/ bmjgh-2020-003936).

Received 9 September 2020 Revised 23 November 2020 Accepted 25 November 2020
A) Check for updates

(c) Author(s) (or their employer(s)) 2020. Re-use permitted under CC BY. Published by BMJ.

For numbered affiliations see end of article.

Correspondence to Dr Mohamed F Jalloh; yum8@cdc.gov

\section{ABSTRACT}

Community engagement and community-based surveillance are essential components of responding to infectious disease outbreaks, but real-time data reporting remains a challenge. In the 2014-2016 Ebola outbreak in Sierra Leone, the Social Mobilisation Action Consortium was formed to scale-up structured, data-driven community engagement. The consortium became operational across all 14 districts and supported an expansive network of 2500 community mobilisers, 6000 faith leaders and 42 partner radio stations. The benefit of a more agile digital reporting system became apparent within few months of implementing paper-based reporting given the need to rapidly use the data to inform the fast-evolving epidemic. In this paper, we aim to document the design, deployment and implementation of a digital reporting system used in six high transmission districts. We highlight lessons learnt from our experience in scaling up the digital reporting system during an unprecedented public health crisis. The lessons learnt from our experience in Sierra Leone have important implications for designing and implementing similar digital reporting systems for community engagement and community-based surveillance during public health emergencies.

\section{BACKGROUND}

The 2014-2016 epidemic of Ebola virus disease (Ebola) in Sierra Leone, Liberia and Guinea remains the largest documented outbreak of Ebola to date. ${ }^{1}$ The epidemic was fuelled by unsafe traditional burials that involved physical contact with corpses and delays in medical care-seeking behaviours that were partly due to the social stigma of Ebola, fear and community distrust in the response. $^{2-4}$ Building and sustain trusting with communities was necessary to rapidly change traditional burial practices and health-seeking behaviours. ${ }^{5-9}$

\section{Summary box}

- Large-scale, localised community engagement was necessary to directly involve communities in the Ebola response and rapidly change traditional burial practices and health-seeking behaviour. Community engagement and community-based surveillance were digitally integrated and scaled-up in six high transmission districts in Sierra Leone.

- Paper-based reporting posed considerable logistical challenges in monitoring community engagement activities, getting community feedback, and reporting suspected cases and deaths in communities.

- The experience of implementing the digital reporting system shed light on the following lessons: (1) technological tools should be driven by the real data needs for epidemic control; (2) the people and users of the technology should be put at the centre and not be overshowed by the technology; (3) instead of trying to design a perfect system, it is more useful to build by doing so that you can turn your challenges into learning opportunities; (4) data collection should be integrated as a key ingredient of structured community engagement (not as a standalone activity), and it should complement and inform interaction between communities and trusted interlocutors; (5) increased financial investments are needed to maximise the benefit of a digital reporting system to translate community-based data into action; (6) data reporting synergies are possible at the community level despite missed opportunities for formal data integration.

- The lessons we learnt in transitioning to a digital data collection and reporting system for community engagement and community-based surveillance have implications for responding to other health emergencies.

In Sierra Leone, the social mobilisation pillar was established in June 2014 to coordinate and provide strategic oversight of 
community engagement activities across all 14 districts. ${ }^{10}$ The Social Mobilisation Action Consortium (SMAC) was formed in September 2014 to support the pillar in scaling-up structured, data-driven community engagement. ${ }^{11}$ As part of the consortium, GOAL Ireland and Restless Development recruited, trained and supported 2500 community mobilisers to implement Community-led Ebola Action planning. ${ }^{12}$ FOCUS 1000, a local non-governmental organisation, engaged almost 6000 religious leaders to promote Ebola prevention practices, especially safe burial measures. ${ }^{13}$ BBC Media Action supported 42 local radio stations to improve the quality of Ebola risk communication through various radio programmes. ${ }^{14}$

\section{CROSS-CUTTING CHALLENGES WITH PAPER-BASED REPORTING}

Digital data collection was not common in Sierra Leone prior to the Ebola outbreak. National surveys and census were done using paper-based means. ${ }^{15} 16$ Cross-cutting logistical challenges with paper-based reporting during the response included time spent on verifying and understanding the handwritten forms as well as additional time for data entry, cleaning and processing. ${ }^{17}$ Although paper-based reporting tools were initially used by community mobilisers to a considerable degree of success, over time, cross-cutting challenges were also experienced in deploying the paper-based tools across all partner organisations. ${ }^{11}$

\section{INTERVENTION-SPECIFIC CHALLENGES WITH PAPER-BASED REPORTINGNING FROM PAPER-BASED TO DIGITAL DATA}

Establishing a functional paper-based reporting for religious leaders would have required a separate category of paid religious leaders or additional SMAC staff to physically travel to collect the weekly reporting forms. Another alternative was to provide the trained religious leaders with transportation allowance to travel to a central location on a weekly basis to submit their reports to SMAC staff. Both options would have significantly increased the cost of the religious leader intervention, added time to obtain the reports, and posed additional data quality issues. SMAC partner radio stations also initially lacked a functional way to systematically report their weekly radio programming activities.

\section{TRANSITIONING FROM PAPER-BASED TO DIGITAL DATA COLLECTION}

In January 2015, SMAC received a grant from the Bill \& Melinda Gates Foundation to transition from paper-based to digital reporting in six prioritised high transmission districts (Kambia, Kono, Moyamba, Port Loko, Western Area Rural, Western Area Urban). ${ }^{18}$ The timeline of the system's rapid design and deployment is outlined in table 1 . The consortium established a Monitoring and Evaluation Working Group to oversee the deployment of the Digital Data Collection System (DDCS). The Working Group together with 10 fulltime staff trained a group of 30 principal trainers using a standard curriculum (online supplemental material). By April 2015, there were 1400 phones registered on the DDCS and 2800 persons trained on the system (table 2). The number of actively registered phones on the DDCS fluctuated over time and reached up to 1500 by June 2015 .

\section{SYSTEM DESCRIPTION}

The DDCS comprised daily and weekly reporting mechanisms using Android-based smart phones (figure 1). Daily reporting consisted of using an interactive short message service (SMS) to send real-time text message alerts of sick people that needed ambulance services and deaths in the community that required safe burials. The Textit platform (Rwanda: Nyaruka and UNICEF) was

Table 1 Timeline of outputs for deploying the digital reporting system, Social Mobilisation Action Consortium (SMAC), Sierra Leone, January-March 2015

\begin{tabular}{|c|c|c|}
\hline \multirow[b]{2}{*}{ Outputs } & \multicolumn{2}{|c|}{ Timelines (2015) } \\
\hline & Planned & Actual \\
\hline $\begin{array}{l}\text { Project management and information technology infrastructure established to oversee rapid } \\
\text { data collection, action triggering, and repository building }\end{array}$ & 15 January & 10 February \\
\hline Data management staff recruited and trained & 31 January & 10 February \\
\hline Data analysis and reporting plan finalised & 31 January & 10 February \\
\hline ODK and SMS data collection platforms customised and tested & 2 February & 10 February \\
\hline Training materials developed and finalised & 4 February & 10 February \\
\hline $\begin{array}{l}\text { DDCS deployed in Western Area Urban and Western Area Rural districts where Ebola } \\
\text { transmission was highest at the time }\end{array}$ & 15 February & 31 March \\
\hline DDCS deployed in remaining four high transmission districts & 22 February & 21 March \\
\hline $\begin{array}{l}\text { SMAC community-based EVD surveillance and reporting initiated and operational in all six } \\
\text { high transmission districts }\end{array}$ & 28 February & 23 March \\
\hline
\end{tabular}

DDCS, Digital Data Collection System; EVD, Ebola virus disease; ODK, Open Data Kit; SMS, short message service. 
Table 2 Distribution of formally trained community reporters and registered phones on the Social Mobilisation Action Consortium's digital data collection system by April 2015

\begin{tabular}{llllrr}
\hline & & & FOCUS & \\
& GOAL Ireland & Restless development & $\mathbf{1 0 0 0}$ & BBC media action & Total \\
\hline \# of community mobilisers trained & 1038 & 526 & $\mathrm{n} / \mathrm{a}$ & $\mathrm{n} / \mathrm{a}$ & 1564 \\
\# of religious leaders trained & $\mathrm{n} / \mathrm{a}$ & $\mathrm{n} / \mathrm{a}$ & 1016 & $\mathrm{n} / \mathrm{a}$ & 1016 \\
\# of radio station managers trained & $\mathrm{n} / \mathrm{a}$ & $\mathrm{n} / \mathrm{a}$ & $\mathrm{n} / \mathrm{a}$ & 202 & 202 \\
Total \# of trained community reporters & 1038 & 526 & 1016 & 202 & 110 \\
Total \# of phones registered on DDCS & 519 & 263 & 508 & 1400 \\
\hline
\end{tabular}

\#, number; DDCS, Digital Data Collection System; n/a, not applicable.

used for the real-time SMS reporting. ${ }^{19}$ SMS alerts were channelled to liaison officers that were integrated into the district-level response. The liaison officers confirmed further reported information before connecting with the district-based burial and surveillance teams to facilitate prompt action.

Weekly reporting consisted of data reporting components on community engagement activities, community feedback and community-based surveillance of sick people and deaths. The Open Data Kit (ODK) platform (www.opendatakit.org) ${ }^{20}$ was used for the weekly reporting. The ODK form was based on an established list of closed-ended and open-ended items initially used by community mobilisers and eventually expanded for use by religious leaders and radio stations. ${ }^{21}$

\section{SUMMARY AND INTERPRETATION OF THE REPORTED DATA}

Between 30 March and 31 December 2015: 1110 alerts of sick people that needed ambulance services and 2978 alerts of deaths that needed safe burial services were reported in real-time through the SMS platform (figure 2). During this period, the number of alerts submitted regarding sick people was highest in May 2015 and the number of death alerts were highest in June 2015. Between 26 March and 31 December 2015: 36619 weekly reports on activities and community feedback were submitted via the ODK platform (figure 3). The number of weekly reports submitted was highest in June 2015 ( $\mathrm{n}=5818)$, which represented $97 \%$ of the expected 6000 monthly total.

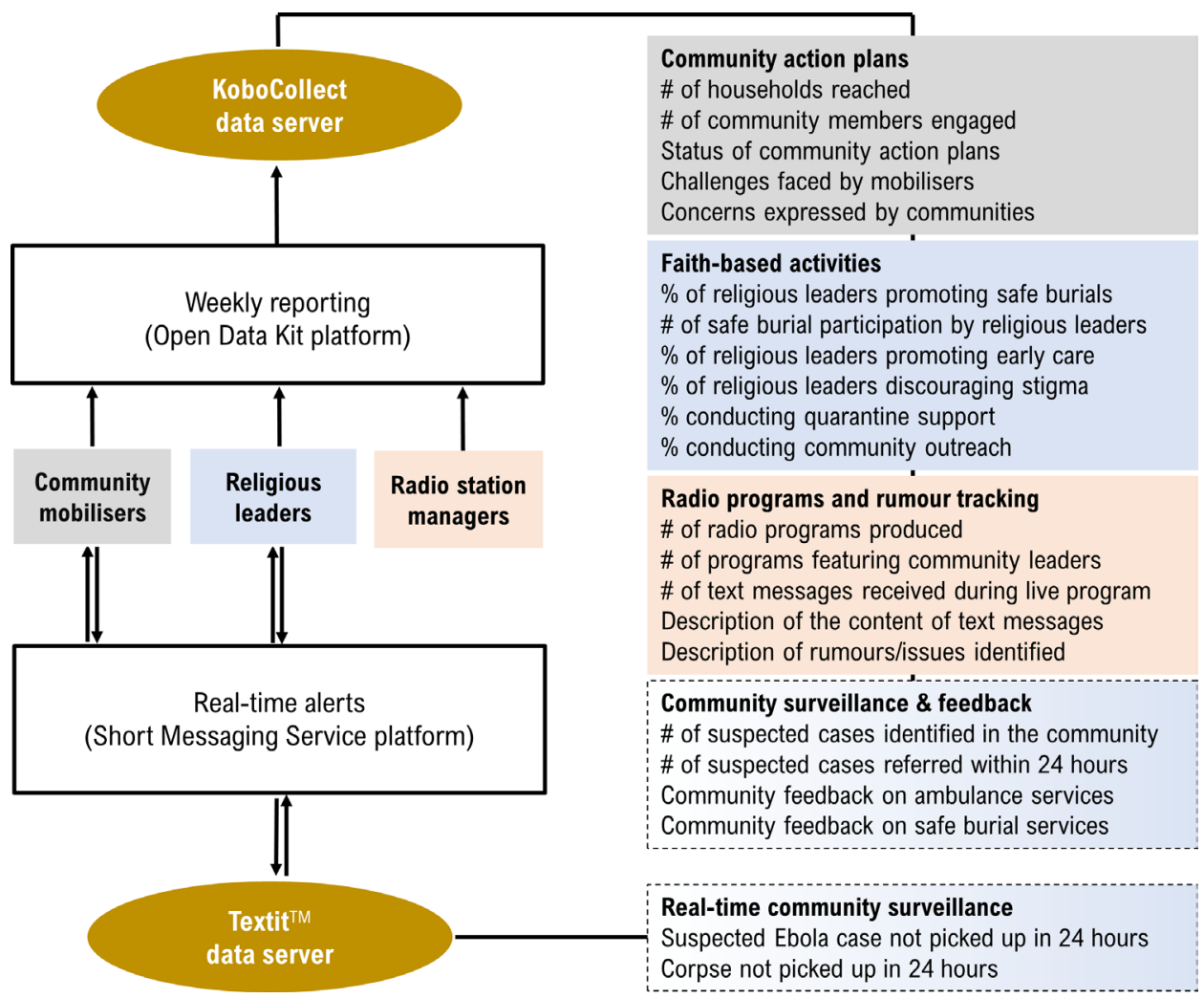

Figure 1 Conceptual framework for the Social Mobilisation Action Consortium (SMAC) 's digital data collection system during the 2014-2016 Ebola outbreak in Sierra Leone. 

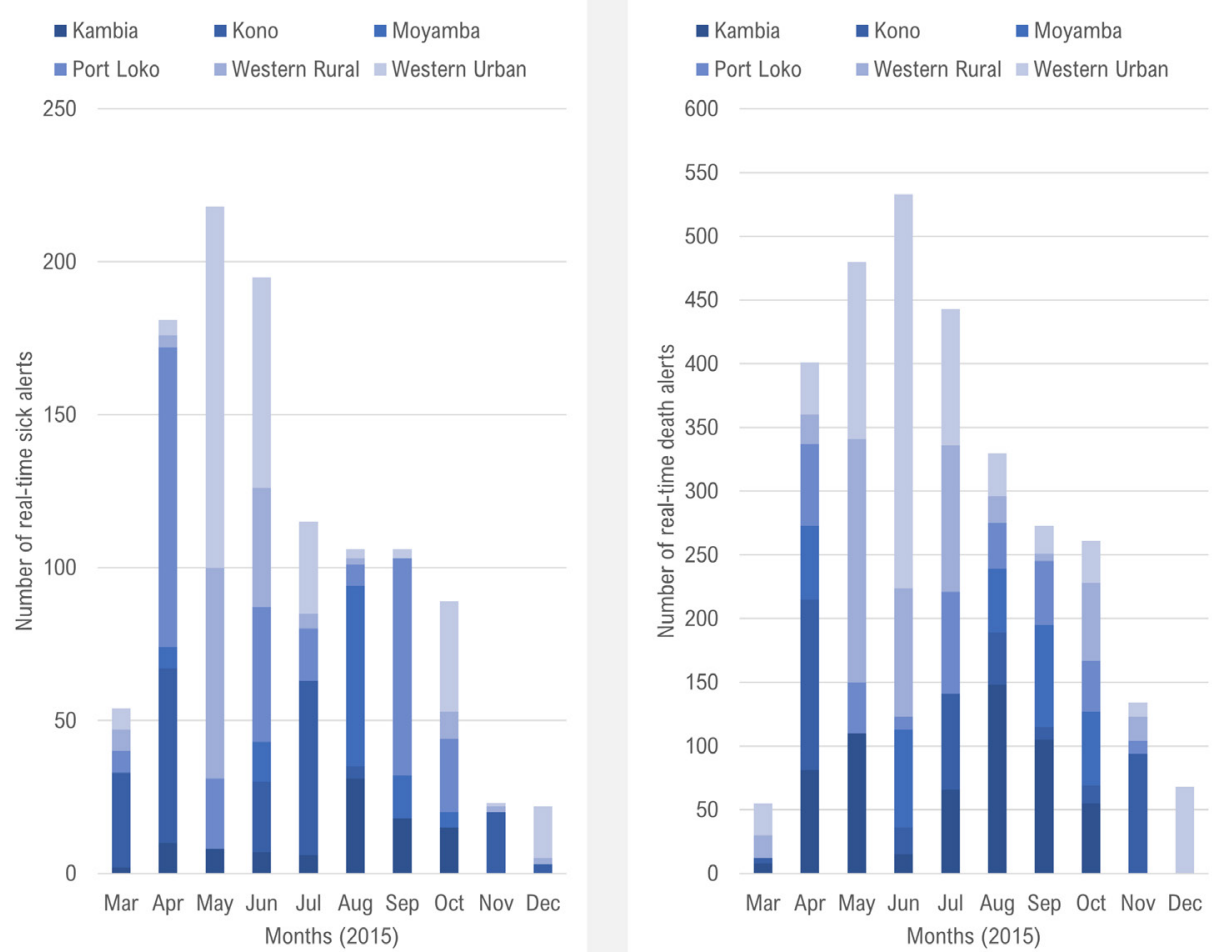

Figure 2 Number of daily real-time alerts on suspected Ebola patients and deaths needing response services submitted through the Textit platform, Social Mobilisation Action Consortium, Sierra Leone, March-December 2015.

Starting in July 2015, the submitted ODK reports and SMS alerts steadily declined until December 2015, which was largely due to lower level of reporting by community mobilisers, religious leaders and radio station managers. The decline in reported alerts should not be necessarily interpreted as commensurate with the decline in the actual numbers of suspected cases and deaths in communities. Nevertheless, the overall waning of the epidemic during this period and the declaration of the end of the epidemic in November 2015 may have influenced the

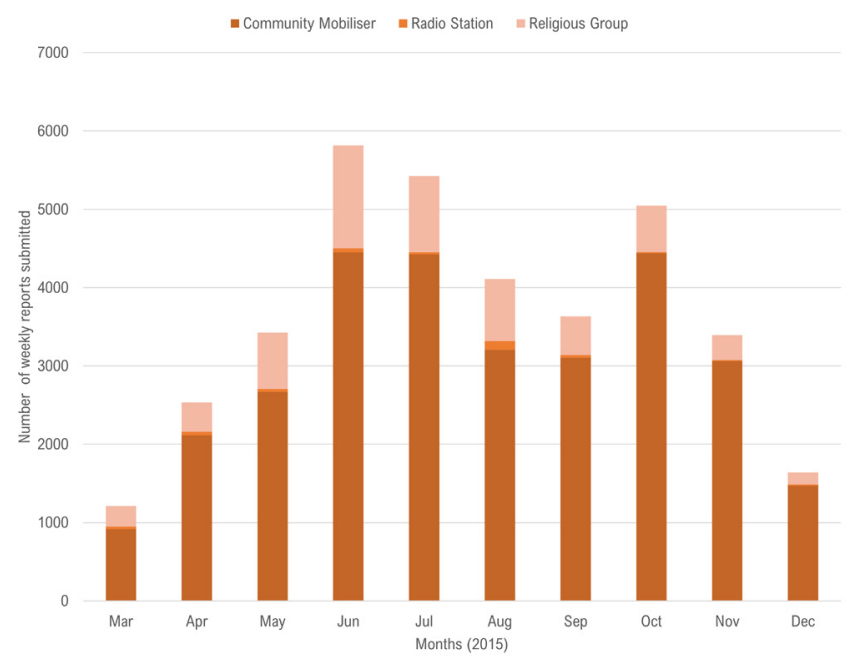

Figure 3 Number of community engagement reports submitted weekly through Open Data Kit platform, Social Mobilisation Action Consortium, Sierra Leone, MarchDecember 2015. reduced level of reporting as volunteers likely became less vigilant in their reporting.

A full cycle of digital reporting via ODK took 10-12 days on average (compared with 12-15 days on average using paper-based tools by mobilisers). The bulk of the time (7 days on average) was spent on the community engagement activities and simultaneous reporting of the data at the end of the week; followed by 3-5 days for data processing and analysis (compared with up to 8 days using paper-based tools). On the other hand, SMS alerts of suspected cases and deaths in the community were transmitted in real-time to liaison officers who usually followed up to gather additional information on the alert within the same day and shared the available alert details with district-based surveillance officers. The merged data from the SMAC intervention have been publicly released ${ }^{21}$ and used in secondary analysis to understand the social and behavioural dynamics of the Ebola epidemic. ${ }^{22}$

\section{WHAT DID WE LEARN?}

\section{Technological tools should be driven by the real data needs} for epidemic control

We ensured that the technology did not drive the process. Instead, the identified data needs and context steered the decisions made about the technological platforms that were leveraged to address our priorities. We aligned the strengths of the technological platforms with the data needed to inform the response. For example, Textit was suitable for sending the alerts on sick people and deaths 
in communities because it was SMS-based and allowed real-time interactive reporting. The direct Short Message Peer-to-Peer (SMPP) connection with the telecommunication company was important to enable large volumes of SMS. For the weekly reporting, we needed a platform that could handle the large quantum of data that the SMAC was collecting to monitor community engagement activities and community surveillance of sick people and deaths to gain insights into important trends over time as the outbreak evolved. A cross-cutting dimension to having the needs drive the process is making a careful determination on the type of data to be collected as well as the scope and frequency of data collection. We learnt that it is important to prioritise the required data and avoid collecting information that may be good to have but not necessary.

\section{Put people at the centre when introducing new technology}

We needed to tailor the digital tools appropriately to our target users. This was partly accomplished by holding user-testing sessions that provided feedback on the system's design for low-literacy users. We ensured that the questions/items in the SMS and ODK platforms were in simple-to-understand language. A dedicated short code number (334) was used to transmit all text messages for ease of reporting. Feedback on the DDCS were regularly solicited through telephone discussions and SMS exchanges between trained community reporters and the data management staff. For instance, in the first 2 months of deploying the system, users reported problem about the irregularity of getting telecommunication connectivity to allow sending of the ODK reports and SMS failures due to disruptions in the SMPP connection. In responding to this feedback, we conducted a troubleshooting activity in May 2015. Our team developed a troubleshooting form to preidentify the range of issues in each district. We recurrently observed that the users changed their configuration settings in the phones, which prevented them from having data access to submit the ODK forms. A month later in June 2015, we then conducted refresher trainings. Both the troubleshooting visits in May and the refresher trainings in June increased reporting levels in those months compared to the prior months. While these large-scale, in-person follow-ups were costly to be done on a monthly basis, similar community-based data reporting efforts should sufficiently budget and plan for monthly follow-up with users to optimise real-time data utilisation.

\section{Do not wait for a perfect system and instead build by doing}

As the system was being rolled out in March 2015, the Government of Sierra Leone instituted a 3-day stay-athome campaign. Trained community mobilisers and religious leaders teamed up with outbreak response teams to conduct house-to-house visits to educate households about Ebola and to actively identify cases during the campaign. The SMS component of the DDCS was rapidly customised to report real-time community feedback on the campaign to the response leadership (online supplemental material). Misinformation and concerns identified were addressed through interactive radio programming and subsequent household visits during the campaign.

Since we lacked experience using the Texit platform, we quickly learnt from UNICEF's use of RapidPro for its UReport, which was built on the same SMS platform as Texit. We learnt from UNICEF about the potential roadblocks when integrating the SMS platform with the telecommunication service providers. With that in mind, we spent a significant amount of time upfront to ensure that we had a stable SMPP integration with the telecommunication providers. Despite efforts to anticipate and plan for this challenge, we ultimately experienced recurring SMPP connection interruptions that sometimes prohibited our users from submitting SMS alerts. We eventually had to migrate to a different telecommunication service provider that was able to provide a more stable connection. We first observed how efficiently the new provider handled the SMPP integration with the Texit platform before deciding on a full migration. While it took nearly 2 months for the first provider to execute the SMPP connection, the second provider did so in just 2 days. We started the migration with 100 users in Western Area to first learn from the experience and then continued with a phased migration of all users.

\section{Data collection by community mobilisers and leaders is not a standalone activity}

Data were collected by community mobilisers, religious leaders and radio station managers as an integrated part of their community engagement roles. ${ }^{12}$ Data collection was not an end in itself. Our primary focus was to understand when and how community mobilisers and religious leaders engaged with local communities. We then carefully integrated key elements of community surveillance with community feedback. The approach aimed to avoid the idea of 'listening in' to communities for the purposes of data collection alone, but of 'listening to' communities whereby the data informed a two-way communication process. Putting digital technology in the hands of community mobilisers and religious leaders to undertake grassroots community surveillance has the advantage of using established community structures and trusted interlocutors to collect data to inform the response. Leveraging community assets and resources for public health surveillance holds tremendous value in responding to future health emergencies, especially when surveillance efforts can be integrated into sustained community engagement.

\section{Increased financial investments are needed to translate community-based data into action}

Nearly US\$600 000 was spent on the digital reporting system between February and December 2015. Start-up costs for procuring the phones plus recurring costs for Internet connectivity and SMS charges comprised the 
largest expense category followed by personnel costs. Excluding start-up costs, the all-inclusive, monthly running cost was approximately US\$32 000 on average between February and December 2015. With the continuous reduction in the market cost of basic smart phones and decreased costs associated with mobile Internet connectivity, it is possible that future implementation of a similar system will incur lower start-up costs.

While a formal cost-benefit analysis is outside the scope of this paper, we should note that it is generally difficult to quantify the benefit of such system in financial terms alone. For instance, the more than 4000 real-time alerts of sick people needing ambulances to isolation centres and deaths needing safe burials represent a qualitative benefit that likely prevented secondary Ebola infections in thousands of households and communities. To the best of our knowledge, the more than 37000 ODK reports submitted through the system represent the single largest community-based data repository available from the Ebola response in Sierra Leone, Liberia and Guinea. Use of ODK for reporting cut the reporting cycle by 3 days on average, which led to timelier availability of information on emerging rumours, challenges, action plans and feedback from communities to inform ongoing response options. Moreover, the system contributed to data-driven decision-making within the consortium as well as in the consortium's advocacy for community-based solutions in the national and sub-national response.

Increased financial investments would have helped to maximise on the benefits of the system including the hiring of dedicated staff focusing on data utilisation. We underestimated the resources needed to rapidly analyse the data and inform national and district level response actions. With the limited staff available, it was difficult to keep up with the quantum of alerts that were generated by the SMAC mobilisers, religious leaders and community members. Although we tried to maximise using the data for response actions through the existing district-based staff such as our district liaison officers, in hindsight, we needed a much larger team of staff to optimise the use of the data. Similar challenges have been documented in the Democratic Republic of Congo regarding the real-time use of sociobehavioural data to inform Ebola epidemic control. ${ }^{23}$ Senior personnel from the respective SMAC partner organisations used the collected data in their day-to-day advocacy and technical assistance efforts to the Government of Sierra Leone, including in their daily participation in the Incident Management meetings at the National Ebola Response Centre.

\section{Data reporting synergies are possible at the community level despite missed opportunities for data integration}

The SMAC's work was not formally linked with the surveillance pillar structures for community event-based surveillance (CEBS), which was in a nascent stage during our DDCS implementation. ${ }^{24}{ }^{25}$ However, at the community level, informal connections were made by SMAC mobilisers and religious leaders with CEBS community monitors. SMAC's liaison officers frequently shared alerts that they received with district surveillance officers, which may have provided duplicate alerts of events reported by CEBS community monitors. Duplicate alerts for the same event may have helped to confirm the event and/or provide additional information that may not have been present in the initial alert received. In some instances, alerts were likely only received from SMAC mobilisers and religious leaders because they had geographic coverage in areas where CEBS was not operational. Looking into the future, the interactive SMS component of the digital system used by SMAC for real-time reporting of key community events may prove helpful in the scaling up of CEBS in Sierra Leone and other similar settings, especially in areas where Internet connectivity remains a challenge.

\section{CONCLUSIONS}

Our digital platforms provided a practical framework for collecting, analysing and reporting data on community engagement and community-based surveillance during the Ebola outbreak in Sierra Leone. Demonstrating the utility of the combined data, including the paper-based data, gave community engagement stakeholders a valuable seat at the table in the Ebola response in Sierra Leone. Since our implementation of the digital system during the Ebola epidemic in Sierra Leone, there has been considerable advancements in community-based digital reporting in outbreak contexts. ${ }^{26}$

However, the lessons learnt from our experience in Sierra Leone and the underlying data could be further leveraged to inform ongoing and future health emergencies. Implementation of similar systems should make considerable provision for real-time data use by having dedicated teams focusing on data utilisation. Communitylevel data collection efforts during health emergencies need to anticipate and plan for not just how to speed up data collection through digitalisation, but also how to improve data quality and use the data to inform response strategies. Given the increasing recognition of the value of community-level data during health emergencies, coordination is required to ensure harmonisation and interoperability of community-based data systems. Emerging digital tools offer unique opportunities to catalyse the timely collection of community feedback and monitoring of community-level behaviours during health emergencies. These digital tools should not be standalone and must be integrated into well-planned community engagement that enable response stakeholders to listen to and partner with communities.

As learnt from the 2014-2016 Ebola epidemic and more recent health emergencies including the COVID-19 pandemic, there is a continued need for increased financial investments to scale-up and integrate community-based data collection, analysis and synthesis into timely strategies and actions for epidemic control. 


\section{Author affiliations}

${ }^{1}$ Department of Global Public Health, Karolinska Institutet, Stockholm, Sweden

${ }^{2}$ Focus 1000 , Freetown, Sierra Leone

${ }^{3}$ Restless Development Sierra Leone, Freetown, Sierra Leone

${ }^{4} \mathrm{GOAL}$, Freetown, Sierra Leone

${ }^{5} \mathrm{BBC}$ Media Action, Freetown, Sierra Leone

${ }^{6}$ Health Education Division, Sierra Leone Ministry of Health and Sanitation, Freetown, Western Area, Sierra Leone

Acknowledgements The authors thank the thousands of community mobilisers, religious leaders, radio station managers and community members who worked with the SMAC during the 2014-2016 Ebola epidemic in Sierra Leone.

Contributors MFJ, PS and MBJ conceptualised the project with inputs from JB, $\mathrm{SB}, \mathrm{KO}, \mathrm{AO}$ and MS. Implementation was led by MFJ, PS, NJ and SAP. MFJ led the writing of the manuscript with contributions from all coauthors. The final version of the manuscript was reviewed and approved by all coauthors.

Funding The SMAC Digital Data Collection System was funded by Bill \& Melinda Gates Foundation (OPP1126040).

Competing interests None declared.

Patient consent for publication Not required.

Provenance and peer review Not commissioned; externally peer reviewed.

Data availability statement Data are available in a public, open access repository. https://doi.org/10.6084/m9.figshare.8247002.v1

Supplemental material This content has been supplied by the author(s). It has not been vetted by BMJ Publishing Group Limited (BMJ) and may not have been peer-reviewed. Any opinions or recommendations discussed are solely those of the author(s) and are not endorsed by BMJ. BMJ disclaims all liability and responsibility arising from any reliance placed on the content. Where the content includes any translated material, BMJ does not warrant the accuracy and reliability of the translations (including but not limited to local regulations, clinical guidelines, terminology, drug names and drug dosages), and is not responsible for any error and/or omissions arising from translation and adaptation or otherwise

Open access This is an open access article distributed in accordance with the Creative Commons Attribution 4.0 Unported (CC BY 4.0) license, which permits others to copy, redistribute, remix, transform and build upon this work for any purpose, provided the original work is properly cited, a link to the licence is given, and indication of whether changes were made. See: https://creativecommons.org/ licenses/by/4.0/.

\section{ORCID iD}

Mohamed F Jalloh http://orcid.org/0000-0002-7206-8042

\section{REFERENCES}

1 World Health Organization. Ebola situation report, 2016. Available: http://apps.who.int/iris/bitstream/10665/204714/1/ebolasitrep_ 30mar2016_eng.pdf [Accessed 19 Mar 2018].

2 Tiffany A, Dalziel BD, Kagume Njenge H, et al. Estimating the number of secondary Ebola cases resulting from an unsafe burial and risk factors for transmission during the West Africa Ebola epidemic. PLoS Negl Trop Dis 2017;11:e0005491.

3 Nuriddin A, Jalloh MF, Meyer E, et al. Trust, fear, stigma and disruptions: community perceptions and experiences during periods of low but ongoing transmission of Ebola virus disease in Sierra Leone, 2015. BMJ Glob Health 2018:3:e000410.

4 Richards P, Amara J, Ferme MC, et al. Social pathways for Ebola virus disease in rural Sierra Leone, and some implications for containment. PLoS Negl Trop Dis 2015;9:e0003567.

5 Gillespie AM, Obregon R, El Asawi R, et al. Social mobilization and community engagement central to the Ebola response in West Africa: lessons for future public health emergencies. Glob Health Sci Pract 2016;4:626-46.
6 Bedrosian SR, Young CE, Smith LA, et al. Lessons of Risk Communication and Health Promotion - West Africa and United States. MMWR Supp/ 2016;65:68-74

7 Government of Sierra Leone. Lessons from the response to the Ebola virus disease outbreak in Sierra Leone, may 2014-November 2015. Freetown: National Ebola Response Center, 2015.

8 Pronyk P, Rogers B, Lee S, et al. The effect of community-based prevention and care on Ebola transmission in Sierra Leone. Am J Public Health 2016;106:727-32.

9 Jalloh MF, Sengeh P, Bunnell RE, et al. Evidence of behaviour change during an Ebola virus disease outbreak, Sierra Leone. Bull World Health Organ 2020;98:330-40.

10 Pedi D, Gillespie A, Bedson J, et al. The development of standard operating procedures for social mobilization and community engagement in Sierra Leone during the West Africa Ebola outbreak of 2014-2015. J Health Commun 2017;22:39-50.

11 Bedson J, Jalloh MF, Pedi D, et al. Community engagement in outbreak response: lessons from the 2014-2016 Ebola outbreak in Sierra Leone. BMJ Glob Health 2020;5:e002145.

12 Restless Development. Community-led Ebola action planning, 2014. Available: http://restlessdevelopment.org/file/smac-clea-fieldmanual-pdf [Accessed 1 Mar 2018].

13 FOCUS 1000. Facilitators guide on training of religious leaders on Ebola prevention and control, 2014. Available: http://focus1000.org/ index.php/downloads-resources/summary/11-social-mobilisation/ 26-facilitators-guide-on-training-of-religious-leaders-on-ebolaprevention-and-control [Accessed 1 Mar 2018].

14 BBC Media Action. Using media and communication to respond to public health emergencies: lessons learned from Ebola, 2015. Available: http://downloads.bbc.co.uk/mediaaction/pdf/ practicebriefings/ebola-lessons-learned.pdf [Accessed 1 Mar 2018].

15 Government of Sierra Leone. Sierra Leone 2004 population and housing census. Freetown: Statistics Sierra Leone, 2004.

16 Statistics Sierra Leone. Demographic and health survey, 2013. Available: https://dhsprogram.com/pubs/pdf/PR42/PR42.pdf [Accessed 1 Mar 2018].

17 Cori A, Donnelly CA, Dorigatti I, et al. Key data for outbreak evaluation: building on the Ebola experience. Philos Trans $R$ Soc Lond B Biol Sci 2017;372:372.

18 Bill and Melinda Gates Foundation. Grant to support the development and implementation of community-designed plans to end transmission of Ebola virus, 2014. Available: https://www. gatesfoundation.org/How-We-Work/Quick-Links/Grants-Database/ Grants/2014/12/OPP1126040 [Accessed 10 Jun 2020].

19 Nyaruka. Textit, 2012. Available: https://textit.in/ [Accessed 20 Jun 2020].

20 OpenDataKit. ODK: tools for the common case, 2019. Available: https://opendatakit.org/software [Accessed 1 Apr 2017].

21 Social Mobilization Action Consortium. Dataset: community engagement data from the 2014-2016 Sierra Leone Ebola outbreak, 2019. Available: https://doi.org/10.6084/m9.figshare.8247002.v1 [Accessed 10 Jun 2020].

22 Skrip LA, Bedson J, Abramowitz S, et al. Unmet needs and behaviour during the Ebola response in Sierra Leone: a retrospective, mixed-methods analysis of community feedback from the social mobilization action Consortium. Lancet Planet Health 2020;4:e74-85

23 Social Science in Humanitarian Action Platform. Ebola outbreak in North Kivu. DRC: Behavioural data compilations, 2020. https://www. socialscienceinaction.org/emergency/ebola-outbreak-drc-2019-20/\# behaviouraldatacompilations

24 Crowe S, Hertz D, Maenner M, et al. A plan for community eventbased surveillance to reduce Ebola transmission - Sierra Leone, 2014-2015. MMWR Morb Mortal Wkly Rep 2015;64:70-3.

25 Ratnayake R, Crowe SJ, Jasperse J, et al. Assessment of community Event-Based surveillance for Ebola virus disease, Sierra Leone, 2015. Emerg Infect Dis 2016;22:1431-7.

26 Centers for Disease Control and Prevention. Guide to global digital tools for COVID-19 response, 2020. Available: https://www.cdc.gov/ coronavirus/2019-ncov/global-covid-19/compare-digital-tools.html [Accessed 29 Oct 2020]. 